\title{
A Pilot Study on Characteristics of Metabolomics and Lipidomics according to Sasang Constitution
}

\author{
Min Jung Kim, ${ }^{1}$ Da-Hye Lee, ${ }^{1,2}$ Jiyun Ahn, ${ }^{1,2}$ Tae-Youl Ha, ${ }^{1,2}$ Young Jin Jang, \\ Eunju Do, ${ }^{3}$ and Chang Hwa Jung $\mathbb{1}^{1,2}$ \\ ${ }^{1}$ Division of Nutrition and Metabolism Research, Korea Food Research Institute, Seongnam, Republic of Korea \\ ${ }^{2}$ Department of Food Biotechnology, Korea University of Science and Technology, Seongnam, Republic of Korea \\ ${ }^{3}$ Clinical Trial Convergence Commercialization Team, Korean Medicine Industry Support Center, Daegu Technopark, \\ Suseong-gu, Daegu, Republic of Korea
}

Correspondence should be addressed to Chang Hwa Jung; chjung@kfri.re.kr

Received 27 July 2017; Revised 16 April 2018; Accepted 12 May 2018; Published 30 May 2018

Academic Editor: Mohammed S. Ali-Shtayeh

Copyright (C) 2018 Min Jung Kim et al. This is an open access article distributed under the Creative Commons Attribution License, which permits unrestricted use, distribution, and reproduction in any medium, provided the original work is properly cited.

\begin{abstract}
Although classification of an individual's Sasang constitution is a key step in the prescription of traditional Korean medicine, the classifying process is complex and not objective. Identification of metabolic-based biomarkers could allow the development of a reliable and sensitive classification technique and even therapeutic management. Our pilot study investigated whether metabolites in plasma are characteristic of Sasang constitutions. Ultra-high-performance liquid chromatography-quadrupole time-of-flight mass spectrometry-based metabolic analysis was conducted against 15 Soyangin (SY), 15 Taeeumin (TE), and 18 Soeumin (SE) individuals, as classified according to the Questionnaire for Sasang Constitution Classification II (QSCC II) and specialist diagnosis. Metabolomics data showed that the TE group was significantly separated from the SY and SE groups. Nine canonical pathways related to constitution; phenylalanine metabolism, aminoacyl-tRNA, tyrosine, and tryptophan biosynthesis were activated in the TE group as compared with the other groups. Similar to the results of the metabolomics analysis, the TE group was also significantly separated from the other two groups by lipidomic analysis. On the other hand, the intensity of lipid metabolites was higher in the SY group than in the other groups. Our findings suggest that the combined analysis of metabolomics and lipidomics can provide useful information for characteristics of Sasang constitutions.
\end{abstract}

\section{Introduction}

Since the results of the Human Genome Project were made known in June 2000, the promise of personalized medicine has been actively pursued; however, this remains in its infancy and there is consensus that further research is needed before personalized medicine becomes an actuality. Although oriental medicine cannot be regarded as personalized medicine, it can be regarded as a group-customized medicine, because it does not treat a condition in the same way across the board, but treatments differ according to the constitutional group to which the patient belongs [1]. Oriental constitutional medicine provides a way to balance human body and mind according to the constitution, not only for the treatment of diseases but also for healthy living [2].
Constitutional medicine in Korea is called Sasang. Sasang constitutional medicine was initiated by Jema Lee (1837-1900) and is the most representative traditional medicine of Korea. It uses an approach of considering how diseases progress differently among different constitutional groups of people and administering treatments based on the recognition of distinct patterns present in each constitutional type [3]. The constitution is classified into four types according to individual's physical and psychological characteristics [4]: Taeyangin (TY), Soyangin (SY), Taeeumin (TE), and Soeumin (SE) [2]. Traditional Korean medicine doctors claim that determination of the constitutional types could be valuable for the prevention and treatment of diseases, because disease susceptibilities and drug responses vary depending on 
the individual's constitution [5], although scientific evidence is still required to verify this claim.

Although the scientific basis of constitutional medicine is still in question, constitution-based oriental medicine is recognized as a medical practice in Korea. However, the Sasang constitution has the disadvantage that it is difficult to diagnose objectively, as the diagnosis of the constitution is made according to the subjective view of the oriental medicine doctor. If a scientific validation method could be developed which allows easy determination of the constitution, it will be highly useful in oriental medicine. Numerous attempts have been made to classify the constitution scientifically; the Questionnaire for Sasang Constitution Classification II (QSCC II) has recently been developed and has been used as a validated, objective assessment [6], although it is continuously being supplemented with additional surveys to improve the accuracy of determination [7]. Questionnaires about the face, body, shape, and personal characteristics have been used in trials of Sasang constitutional diagnosis [8]. Moreover, discrimination of the Sasang constitution, depending on the characteristics of the skin, fingerprints, and voice, has been reported [9-11].

In metabolomics, the causes of life phenomena are systematically identified by analyzing the composition and concentration of metabolic groups that change under various genetic, physiological, or environmental conditions [12]. The emerging field of "metabolomics," in which a large number of small molecules from body fluids or tissues are detected quantitatively in a single step, holds immense potential for early diagnosis, identification of novel drug targets, customized drug treatment, therapy monitoring, and advances in the understanding of the pathogenesis of many diseases [13]. To date, no study has reported the relationship between Sasang constitution and metabolites.

Our study investigated whether blood metabolites differ significantly depending on the Sasang constitution as classified by QSCCII diagnosis. Polar metabolites and lipids were identified using liquid chromatography-mass spectrometry(LC-MS-) based metabolomics and lipidomics. The analysis was performed on only three of the Sasang constitutions, as the TY constitution is very rare.

\section{Materials And Methods}

2.1. Subjects and Study Design. This human study was approved (KMISC-FD-22) by Daegu Oriental Hospital of Daegu Haany University (Daegu, South Korea). Healthy Korean males between 21 and 29 years of age were recruited. Each participant was grouped according to Sasang constitutional types (SCT), using the integrated diagnosis model combining four individual models based on Questionnaire for Sasang Constitutional Classification II (QSCC II), face, body shape, and voice, by oriental medicine doctors (Daegu Haany University Medical Center). Participants with a body mass index $(\mathrm{BMI})<18.5 \mathrm{~kg} / \mathrm{m}^{2}$ or $>30 \mathrm{~kg} / \mathrm{m}^{2}$ [14], hypertension, and ALT and AST greater than twice the normal upper limit or who were participating in any other study or had donated blood during the last 1 month before the start of this study or had preexisting chronic diseases or were taking drugs were excluded. Fifteen TE, 15 SY, and 18 SE individuals were identified. The TY type is extremely rare in the population [15], and no such subjects were classified in our study. The subjects had fasted $10-17 \mathrm{~h}$ prior to collection of blood samples in an EDTA plasma tube. Blood samples were centrifuged at $1550 \times \mathrm{g}$ for $10 \mathrm{~min}$ at $4^{\circ} \mathrm{C}$ to collect the plasma. General characteristics of the participants, such as BMI, waist-to-hip ratio (WHR), visceral fat area (VFA), blood pressure, and plasma lipid profiles, were determined.

2.2. Metabolomics Analysis. Plasma protein was precipitated by addition of cold acetonitrile. After shaking for $30 \mathrm{~min}$ at $4^{\circ} \mathrm{C}$, the samples were centrifuged at $9,000 \times \mathrm{g}$ for $10 \mathrm{~min}$ at $4^{\circ} \mathrm{C}$ and the supernatant was dissolved in $20 \%$ aqueous methanol containing caffeine [16]. The analyses were performed using an ACQUITY Ultra-Performance Liquid Chromatography (UPLC) system (Waters, Milford, MA, USA) coupled to an ultra-performance liquid chromatographyquadrupole time of flight mass spectrometry (UPLCESI/Q-TOF) system (Waters Corp., Manchester, UK) with ACQUITY UPLC BEH C18 $(2.1 \times 100 \mathrm{~mm}, 1.7 \mu \mathrm{m})$ column. The column temperature was conditioned at $30^{\circ} \mathrm{C}$. The flow rate was set at $0.30 \mu \mathrm{l} / \mathrm{min}$. The mobile phase was composed of (A) $0.1 \%$ formic acid aqueous solution and (B) $0.1 \%$ formic acid in acetonitrile, at an injection volume of $5 \mu \mathrm{l}$. The conditions were as follows: initial condition of $98 \%$ A, 0-13 min with $98-5 \% \mathrm{~A}$, and $13-14$ min with $5 \% \mathrm{~A}$ and returning to $98 \%$ A for a 2 min reequilibration step. The mass spectrometer was a Waters SYNAPT G2-Si mass spectrometer with an electrospray ionization (ESI) mode. The ESI source was set in positive and negative ESI mode with a scan range of $\mathrm{m} / \mathrm{z} 50-1,000$. Argon was used as collision gas, and nitrogen was used as desolvation gas. The voltage of capillary, cone, and collision energy was set at $3.0 \mathrm{kV}, 40 \mathrm{~V}$, and $25 \mathrm{~V}$, respectively. The gas flow for desolvation and cone was 800 and $50 \mathrm{~L} / \mathrm{h}$. The source temperature and desolvation gas temperature were 110 and $350^{\circ} \mathrm{C}$, respectively.

2.3. Lipidomics Analysis. The sample extraction is briefly described as follows $[17,18]$. Serum samples $(50 \mu \mathrm{l})$ were extracted with a mixture of chloroform and methanol (1:2; $200 \mu \mathrm{l})$. This solution was then vortexed for $30 \mathrm{~min}$ at room temperature and immediately vortexed. The sample was centrifuged at $13,000 \mathrm{rpm}$ at $4^{\circ} \mathrm{C}$. The supernatant was completely evaporated under a stream of nitrogen. The lipid extract was diluted with an isopropanol:acetonitrile: water $(2: 1: 1, \mathrm{v} / \mathrm{v} / \mathrm{v})$ solution. Lipid extracts were analyzed on a SYNAPT G2-Si mass spectrometer (Waters, Manchester, UK) coupled to an ACQUITY UPLC system (Waters, Milford, MA, USA) with an ACQUITY UPLC CSH C18 $(2.1 \times 100$ $\mathrm{mm}, 1.7 \mu \mathrm{m})$ column. The column temperature was set at $55^{\circ} \mathrm{C}$. Mobile phase A was $10 \mathrm{mM}$ ammonium acetate and $0.1 \%$ formic acid in an acetonitrile: water mixture (60:40, $\mathrm{v} / \mathrm{v})$; mobile phase B was $10 \mathrm{mM}$ ammonium acetate and $0.1 \%$ formic acid in an isopropanol: acetonitrile mixture (90:10, $\mathrm{v} / \mathrm{v})$. The gradient profile was $60-43 \%$ B over $2 \mathrm{~min}, 50 \%$ $\mathrm{B}$ at $2.1 \mathrm{~min}, 54 \% \mathrm{~B}$ at $12 \mathrm{~min}$, and $99 \% \mathrm{~B}$ at $18 \mathrm{~min}$, followed by equilibration at $40 \% \mathrm{~B}$ for $2 \mathrm{~min}$ at a flow rate of $0.4 \mu \mathrm{l} / \mathrm{min}$. The Q-TOF-MS was operated in positive and 
negative electrospray ionization mode within a mass range of $100-1,000 \mathrm{~m} / z$. The source temperature was set at $120^{\circ} \mathrm{C}$, voltage of the sampling cone is $30 \mathrm{~V}$, and the desolvation gas $(800 \mathrm{~L} / \mathrm{h})$ is at $250^{\circ} \mathrm{C}$. The collision energy was ramped from 20 to $35 \mathrm{~V}$ and the capillary voltages were set at $2.0 \mathrm{kV}$ (for positive) and $1.0 \mathrm{kV}$ (for negative). All spectrum data were collected in continuum format using the $\mathrm{MS}^{\mathrm{E}}$ acquisition mode. Mass accuracy was calibrated using sodium format, and leucine enkephalin was used as lock mass.

2.4. Data Processing And Statistical Analysis. The raw data were processed using Progenesis QI data analysis software (Nonlinear Dynamics, Newcastle, UK) for chromatographic alignment, normalization, peak picking, and compound identification. The resulting data sets were imported into SIMCA-P version 12.0.1 (Umetrics, Umeå, Sweden) for multivariate analysis and were mean-centered scaled. Assignment of metabolites contributing to the observed variance was performed using ChemSpider (http://www.chemspider.com), Human Metabolome Database (http://www.hmdb.ca), and LIPID MAPS (http://www.lipidmaps.org). Metabolic pathway analysis was performed using MetaboAnalyst 3.0 (http://www.metaboanalyst.ca), which is based on database sources such as KEGG and HMDB, to identify the metabolic pathways that are affected and to facilitate biological interpretation. One-way analysis for variance (ANOVA) with Tukey's post hoc tests was carried out for the comparison between three groups. Potential biomarkers were selected using VIP > 1 and $P<0.05$.

\section{Results}

3.1. Subjects' Characteristics. Table 1 shows the general characteristics of participants. There were significant differences between the three groups in terms of BMI, body fat percentage (BFP), WHR, VFA, and systolic blood pressure (SBP). Although there was no significant difference between the SY and TE groups, both groups were significantly different from the SE group. SE persons have a more skinny body phenotype than SY and TE persons. This result is consistent with the results of previous studies showing a relatively low BMI for SE people [19]. In addition, total cholesterol (TC) was higher in the TE group than in the SY group; however, there was no significant difference when compared to the SE group. SBP levels were higher in the TE and SY groups than in the SE group. It is known that there may be constitutions among the Sasang constitutions that are likely to become obese and that there are differences in constitutional characteristics according to lifestyle.

3.2. Metabolic Profiles of Plasma Samples. The metabolomic profiles of plasma samples from the three groups (15 TE, 15 SY, and 18 SE) were characterized and compared. A total of 3,000 molecular features in positive and negative modes were obtained and subjected to statistical analysis using Progenesis Q1 and SIMCA-P version 12.0.1 software, including partial least-squares discriminant analysis (PLS-DA). As shown in the PLS-DA score plots, the TE group was significantly separated from the other two groups (Figures 1(a) and $1(\mathrm{c})$ ). On the other hand, there was no significant difference between the SY and SE groups. The PLS-DA score plots between the Sasang constitutions showed a similar pattern in both negative and positive ion modes. The validation plots from the permutation tests strongly supported the validity of the PLS-DA scores (Figures $1(\mathrm{~b})$ and $1(\mathrm{~d})$ ). Validity was supported by the finding that all permuted $\mathrm{R}^{2}$ and $\mathrm{Q}^{2}$ values on the left were lower than the original point on the right and that the $\mathrm{Q}^{2}$ regression line (in blue in the plots in Figure 1) had a negative intercept.

A total of 33 metabolites (VIP $>1, P<0.05$ ) were identified as potential biomarkers for diagnosis of Sasang constitution types (Table 2). Among the 33 metabolites, the intensity of 27 metabolites showed the same tendency in order of TE, SE, and SY groups. In a comparison of the TE and SY groups, a total of 23 metabolites, such as 3-hydroxybutyric acid, leucine, caffeic acid, melatonin, and phenyllactic acid, were significantly higher in the TE than in the SY types. In addition, the levels of 4-hydroxybenzaldehyde, glutamic acid, leucine, indole-3lactic acid, acetylcarnitine, melatonin, and stearoylcarnitine were significantly different between the TE and SE groups. Eight significant metabolites were identified in the comparison of the SY and SE groups. In particular, glutamic acid may be a potential biomarker candidate for the diagnosis of Sasang constitution type. The relative intensity of each metabolite in the sample from each group was shown in heat map (Figure 3(a)) MetaboAnalyst indicates that 11 pathways, such as phenylalanine metabolism; aminoacyl-tRNA biosynthesis; tyrosine and tryptophan biosynthesis; D-glutamine and D-glutamate metabolism; valine, leucine, and isoleucine biosynthesis; and nitrogen metabolism, were regarded as the targeted metabolic pathways (Figure 3(b)). This result suggests that the difference in Sasang constitutions mainly involves differences in amino-acid-related metabolism.

3.3. Lipidomic Profiles of Plasma Samples. SE persons are characterized by having a slender body shape, unlike TE and SY persons [5]. Lipid profiling of individuals with these different constitutions was expected to show a clear trend between the SE and the other two constitutions. The PLSDA score plots showed a significant separation between the TE and the other two constitutions in both positive and negative modes (Figures 2(a) and 2(c)). The validation tests supported the validity of the PLS-DA models (Figures 2(b) and $2(\mathrm{~d})$ ). Although there was no significant difference in the lipid profiles of the SE and SY groups, the significance in the negative and positive modes was 0.077 and 0.06 , respectively. Moreover, we identified 36 lipids (Table 3). Among the 36 metabolites, 16 metabolites, such as FA (20:4), FA (22:5), FA (22:6), LPC (16:1), LPE (16:0), LPI (18:0), PC (32:1), PC (33:1), PC (34:1), 1-deoxysphinganine, sphinganine (d18:1), and sphinganine (d20:1), were significantly grouped among the three groups. In the comparison of the SY and SE groups, 20 fatty acids showed significant differences, which was more than the eight metabolites showing differences in the metabolomics profiles. Our results showed that the lipidomic analysis showed that the SY group was characterized by different lipid profiles as compared with SE group 


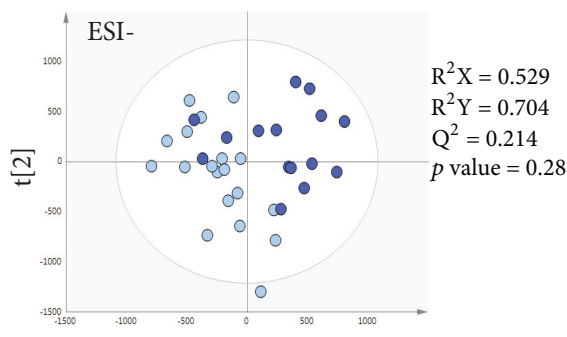

SE

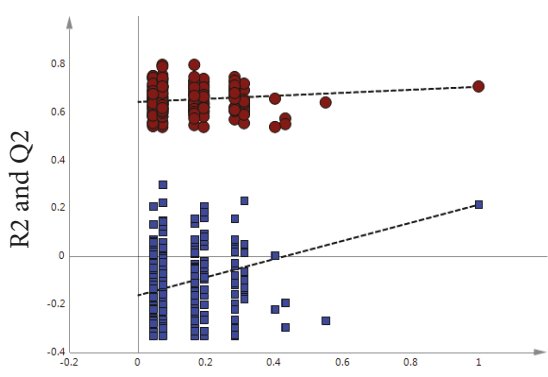

Intercepts: R2=0.641; Q2= -0.164

- $\mathrm{R} 2$

- Q2

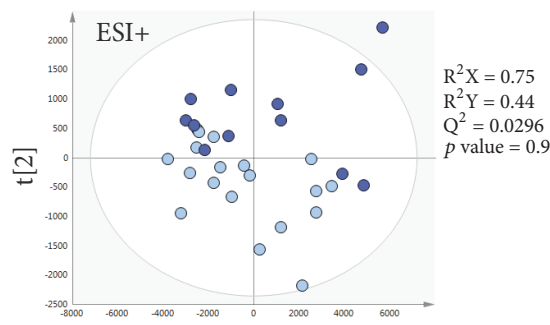

SE

SY

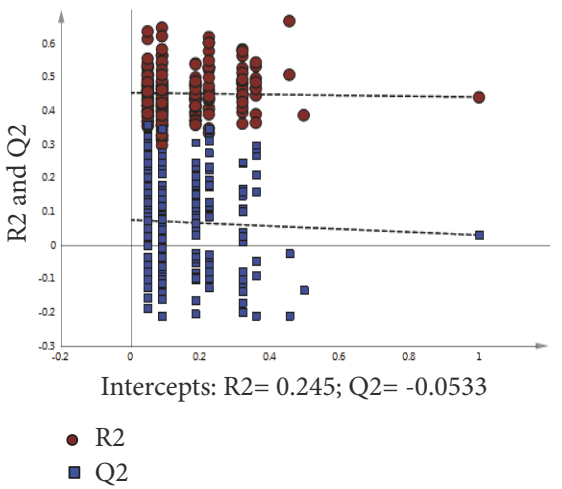

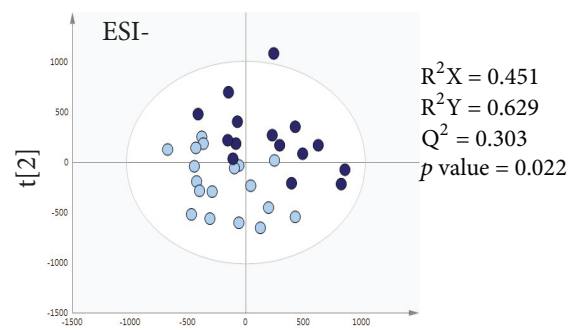

SE

- $\mathrm{TE}$

(a)

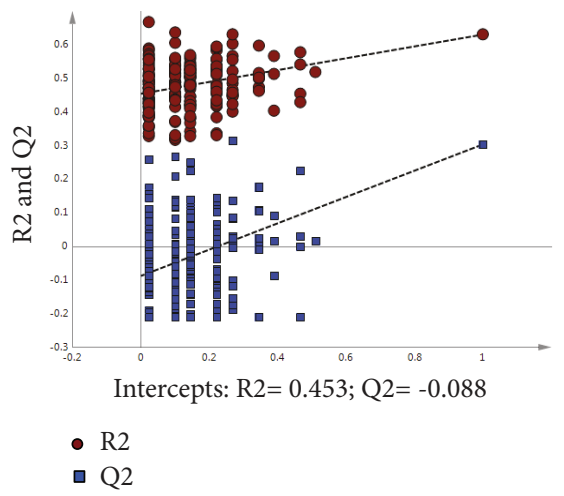

(b)
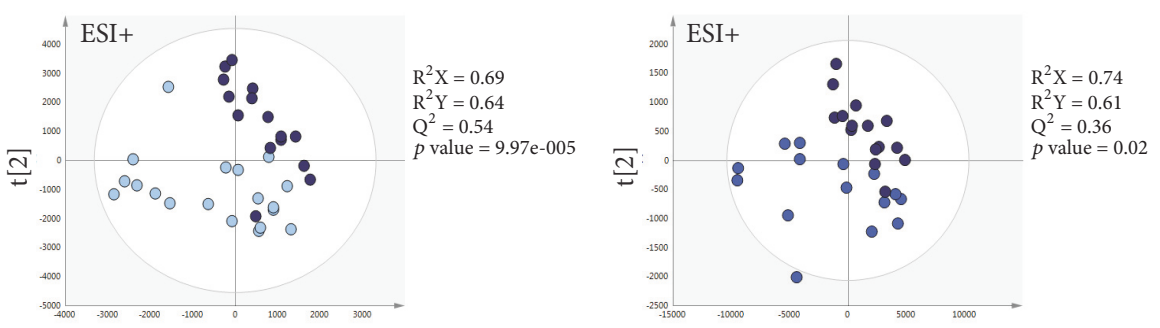

SE

- TE

(c)
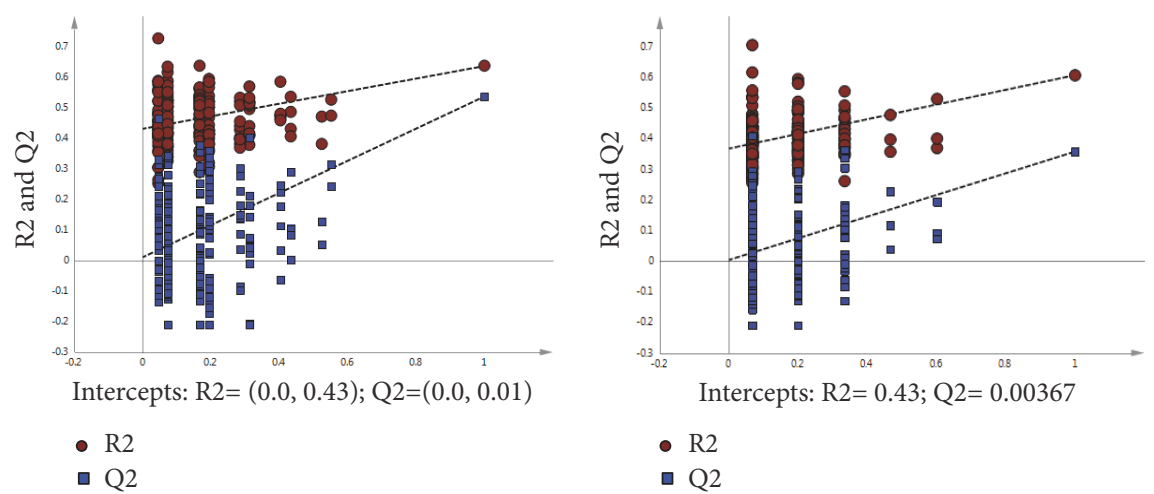

Intercepts: R2=0.43; Q2 $=0.00367$

- $\mathrm{R} 2$

- Q2

(d)

FIGURE 1: Partial least-squares discriminant analysis (PLS-DA) score and permutation results from plasma metabolites of participants classified according to Sasang constitution types based on metabolomics analysis. (a) PLS-DA score plot in ESI- mode. (b) Validation plot in ESI- mode. (c) PLS-DA score plot in ESI+ mode. (d) Validation plot in ESI+ mode. SY: Soyangin; TE: Taeeumin; SE: Soeumin. 

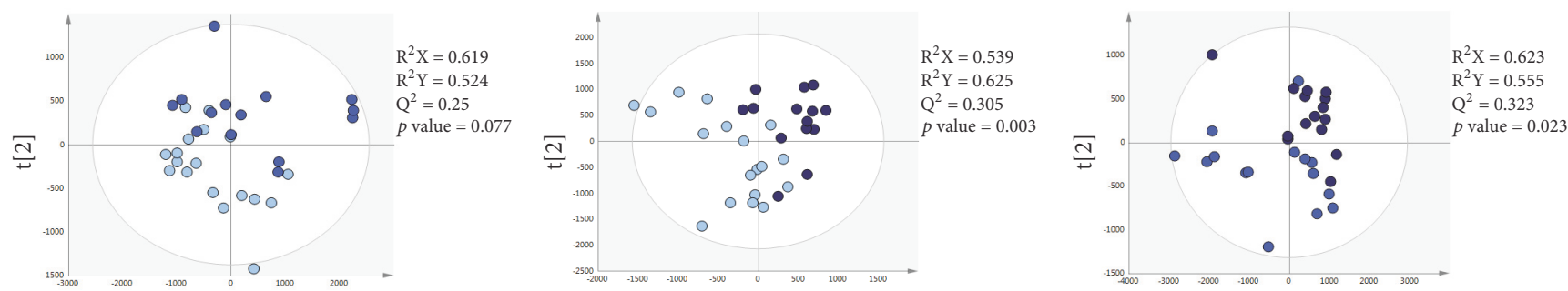

SE

SE

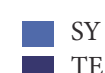

(a)
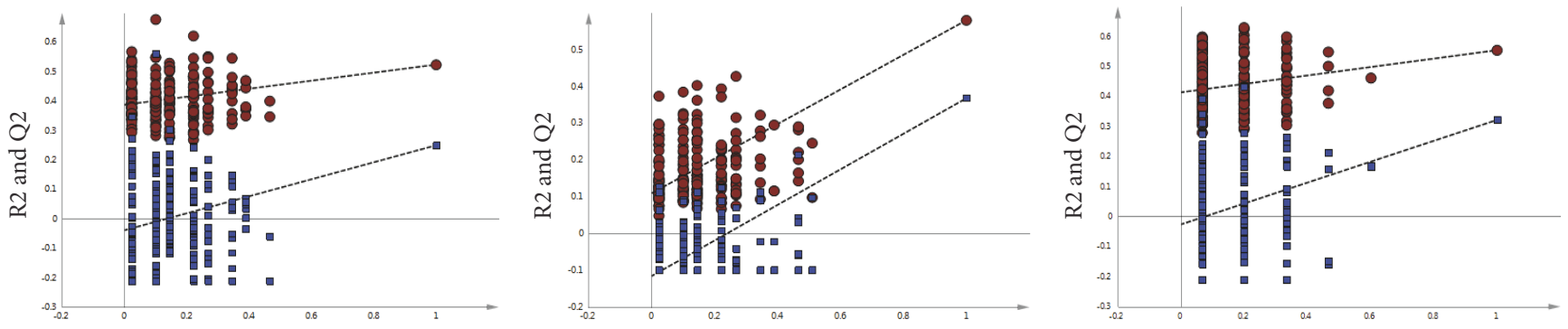

Intercepts: R2=0.388; Q2 $=-0.0387$

- $\mathrm{R} 2$

Intercepts: $\mathrm{R} 2=0.356 ; \mathrm{Q} 2=-0.1$

Intercepts: R2=0.414; Q2 $=-0.10257$

- Q2

- $\mathrm{R} 2$

- R2

- Q2

- Q2

(b)
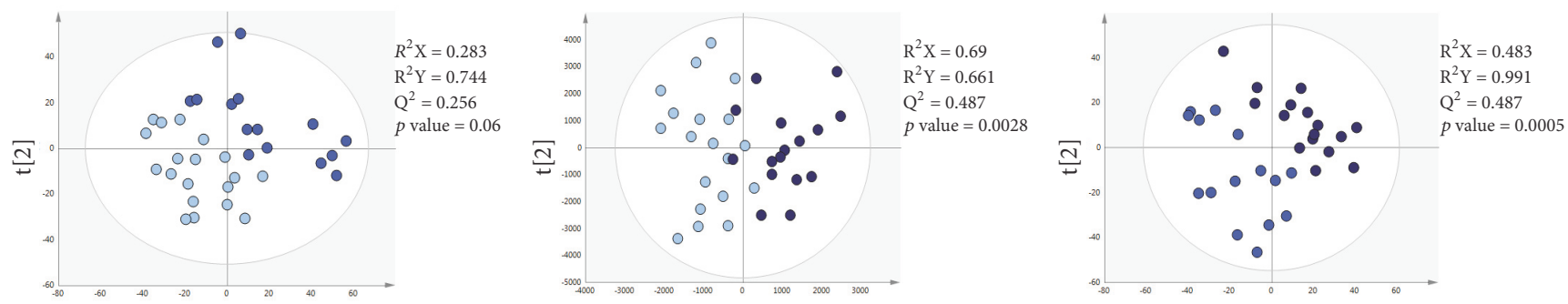

SE

SE

TE

SY

(c)

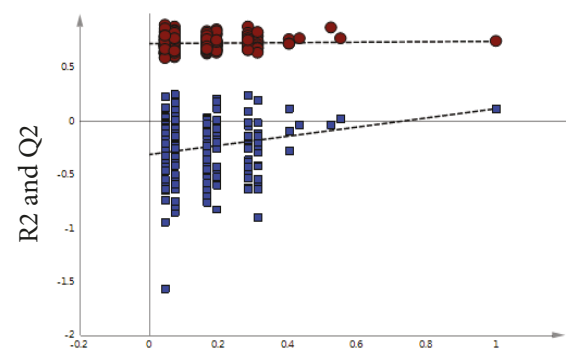

Intercepts: $\mathrm{R} 2=0.725 ; \mathrm{Q} 2=-0.313$

- $\mathrm{R} 2$

- $\mathrm{Q} 2$

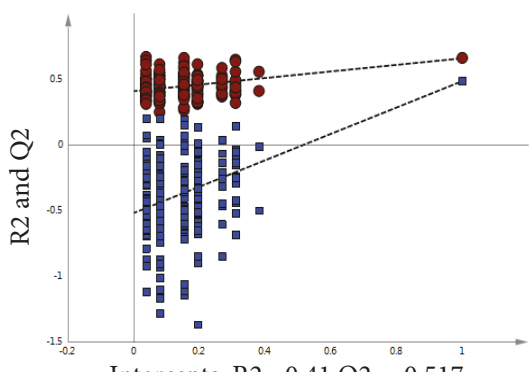

Intercepts: R2=0.41 Q2= -0.517

- $\mathrm{R} 2$

- Q2

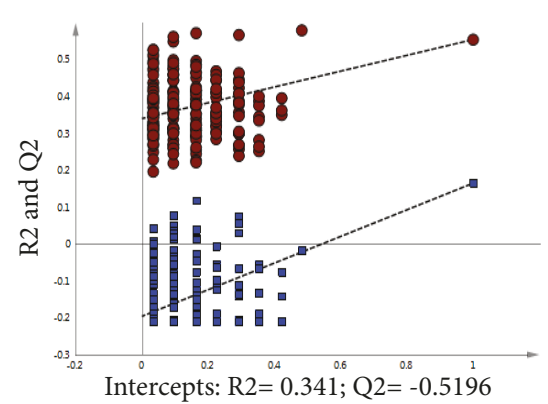

- R2

- Q2

(d)

FIGURE 2: Partial least-squares discriminant analysis (PLS-DA) score and permutation results from plasma metabolites of participants classified according to Sasang constitution types using lipidomics analysis. (a) PLS-DA score plot in ESI- mode. (b) Validation plot in ESImode. (c) PLS-DA score plot in ESI+ mode. (d) Validation plot in ESI+ mode. SY: Soyangin; TE: Taeeumin; SE: Soeumin. 


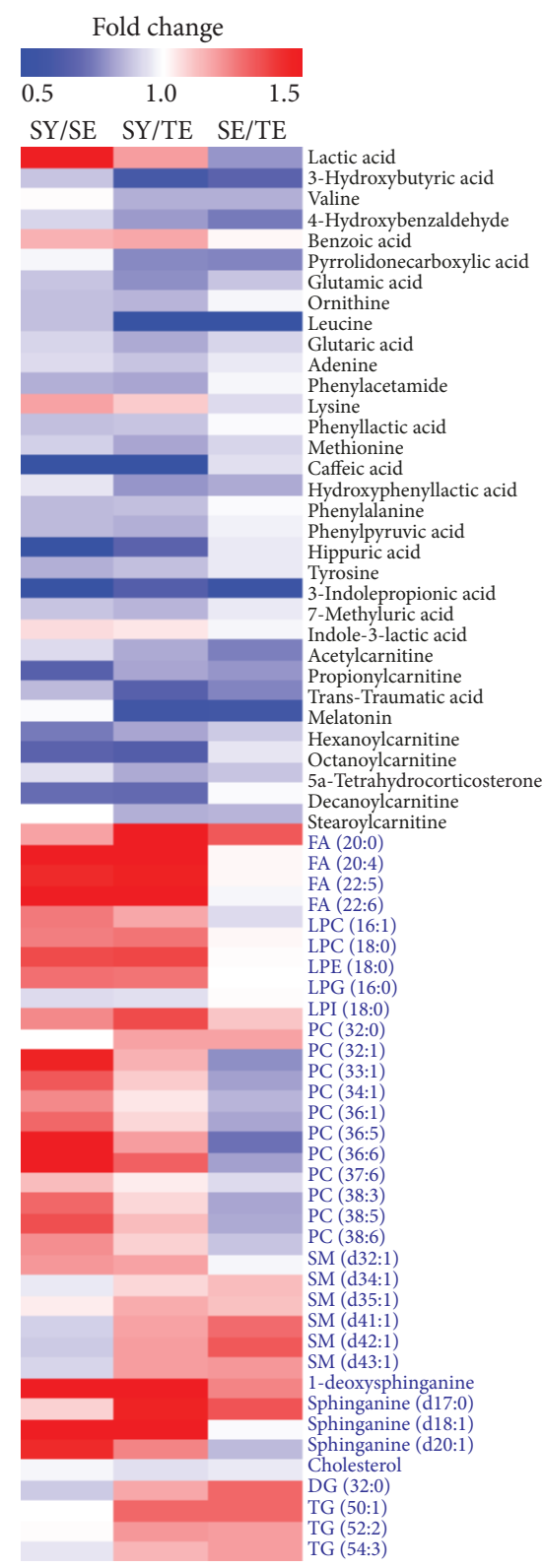

(a)

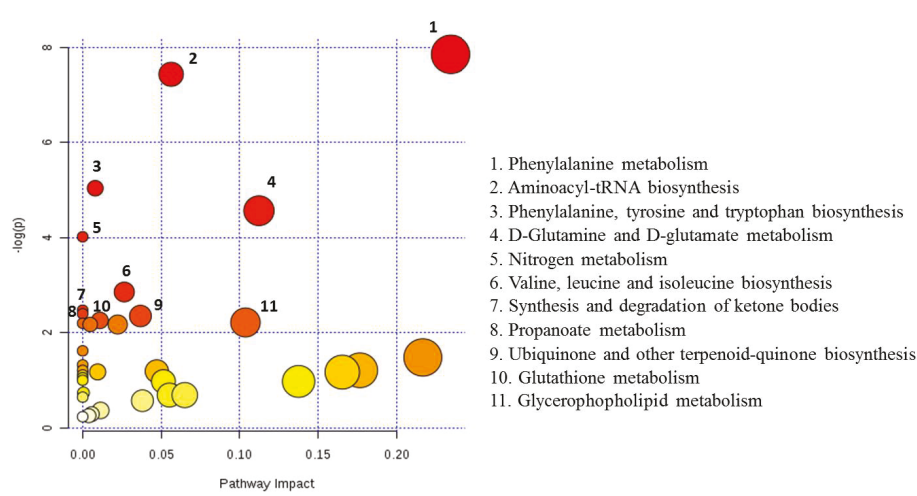

(b)

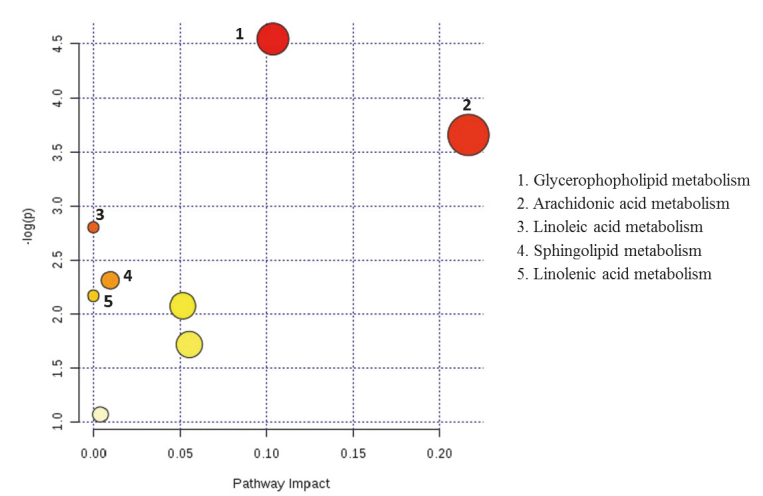

(c)

FIGURE 3: Heat map and metabolic pathway of metabolites identified by metabolomics and lipidomics analysis. (a) Heat map of identified metabolites. (b) Amino acid metabolism. (c) Lipid metabolism. SY: Soyangin; TE: Taeeumin; SE: Soeumin. 
TABLE 1: General characteristics of participants.

\begin{tabular}{|c|c|c|c|c|}
\hline Variable & SY & $\mathrm{TE}$ & SE & $P$ value \\
\hline No. of participants & 15 & 15 & 18 & \\
\hline Age (year) & $24.07 \pm 2.71$ & $24.33 \pm 2.02$ & $23.67 \pm 1.68$ & 0.670 \\
\hline BMI $\left(\mathrm{kg} / \mathrm{m}^{2}\right)$ & $25.09 \pm 2.21^{\mathrm{b}}$ & $25.57 \pm 1.90^{\mathrm{b}}$ & $21.36 \pm 1.75^{\mathrm{a}}$ & $<0.0001^{* * * *}$ \\
\hline BFP (\%) & $22.65 \pm 4.34^{\mathrm{b}}$ & $24.02 \pm 4.06^{\mathrm{b}}$ & $19.08 \pm 4.79^{\mathrm{a}}$ & $0.007^{* *}$ \\
\hline WHR & $0.87 \pm 0.03^{b}$ & $0.86 \pm 0.04^{\mathrm{b}}$ & $0.83 \pm 0.03^{\mathrm{a}}$ & $0.003^{* *}$ \\
\hline VFA & $81.91 \pm 28.34^{\mathrm{b}}$ & $86.55 \pm 19.62^{\mathrm{b}}$ & $60.54 \pm 25.64^{\mathrm{a}}$ & $0.009^{*}$ \\
\hline Glucose (mg/dL) & $92.20 \pm 7.59$ & $90.20 \pm 4.04$ & $91.61 \pm 9.10$ & 0.747 \\
\hline $\mathrm{TC}(\mathrm{mg} / \mathrm{dL})$ & $161.20 \pm 32.74^{\mathrm{a}}$ & $186.60 \pm 26.28^{\mathrm{b}}$ & $168.67 \pm 27.47^{\mathrm{ab}}$ & 0.060 \\
\hline TG (mg/dL) & $126.87 \pm 138.15$ & $111.33 \pm 65.40$ & $74.22 \pm 37.86$ & 0.220 \\
\hline SGOT & $22.73 \pm 6.72^{\mathrm{ab}}$ & $26.60 \pm 11.63^{b}$ & $19.50 \pm 4.42^{\mathrm{a}}$ & $0.048^{*}$ \\
\hline SGPT & $33.87 \pm 11.69$ & $29.80 \pm 13.21$ & $24.39 \pm 9.57$ & 0.069 \\
\hline$\gamma$-GPT & $32.00 \pm 11.59$ & $28.40 \pm 13.28$ & $26.17 \pm 10.10$ & 0.363 \\
\hline Protein (g/dL) & $7.51 \pm 0.26$ & $7.57 \pm 0.27$ & $7.36 \pm 0.30$ & 0.090 \\
\hline Albumin (g/dL) & $4.67 \pm 0.23$ & $4.77 \pm 0.24$ & $4.76 \pm 0.29$ & 0.532 \\
\hline SBP (mmHg) & $127.40 \pm 9.93^{\mathrm{b}}$ & $126.27 \pm 11.51^{\mathrm{b}}$ & $117.56 \pm 10.18^{\mathrm{a}}$ & $0.018^{*}$ \\
\hline DBP (mmHg) & $81.40 \pm 8.77$ & $81.47 \pm 8.59$ & $77.94 \pm 7.77$ & 0.382 \\
\hline $\mathrm{WBC}\left(\times 10^{3} / \mu \mathrm{L}\right)$ & $6.03 \pm 1.46$ & $5.82 \pm 1.32$ & $5.94 \pm 1.55$ & 0.922 \\
\hline $\mathrm{RBC}\left(\times 10^{6} / \mu \mathrm{L}\right)$ & $4.94 \pm 0.43$ & $5.11 \pm 0.35$ & $4.82 \pm 0.40$ & 0.121 \\
\hline $\operatorname{PLT}\left(\times 10^{3} / \mu \mathrm{L}\right)$ & $261.87 \pm 54.80$ & $243.73 \pm 43.89$ & $235.17 \pm 42.61$ & 0.271 \\
\hline
\end{tabular}

SY: Soyangin; TE: Taeeumin; SE: Soeumin; BMI: body mass index; BFP: body-fat percentage; WHR: waist-to-hip ratio; VFA: visceral fat area; TC: total cholesterol; TG: triglycerides; SGOT: serum glutamic oxaloacetic transaminase; SGPT: serum glutamic-pyruvic transaminase; $\gamma$-GPT: gamma-glutamyl transferase; SBP: systolic blood pressure; DBP: diastolic blood pressure; WBC: white blood cell; RBC: red blood cell; PLT: platelet cell. Differences among groups were analyzed using Duncan's multiple range test $(p<0.05)$ and are indicated by different lowercase letters (a, b, and ab). Letter "a" is significant to "b," but "ab" is not significant. $*, * *$, and $* * * *$ denote one-way ANOVA (parametric test).

(Figure 3(a)). Interestingly, MetaboAnalyst analysis showed that there were five canonical pathways that may be related to Sasang constitutions (Figure 3(c)); the top two pathways were glycerophospholipid and arachidonic acid metabolism. In the SY group, these metabolic pathways were more activated than in the SE and TE groups.

\section{Discussion}

This study investigated whether metabolomics and lipidomics analyses of human plasma could classify the Sasang constitution types. Sasang constitutional medicine prescribes medicinal herbs according to the individual type, based on the biopsychosocial perspectives [5]. In terms of personalized medicine, which is a current focus point, it is surprising that such an analysis has not yet been reported. In an effort to develop a method for classifying Sasang constitution type, QSCC II method was developed [8], which could assist practitioners in diagnosing the Sasang constitution type but was limited in terms of scientific evidence $[6,20]$. The accurate classification of Sasang constitution type has been a major concern in both conventional and complementary medicine and could play an important role in oriental medical prescriptions and treatment for certain diseases, such as stroke, diabetes, and metabolic syndrome [15, 21-23].

In this study, we first investigated the use of metabolomics for classifying the Sasang constitution types, as metabolic analysis has the advantage of allowing the prediction or diagnosis of human diseases, such as cancer, diabetes, and chronic liver disease [13, 24-26]. Our results showed the possibility of classifying Sasang constitution types by analyzing both metabolomics and lipidomics. The TE group was significantly separated from the SE and SY groups by metabolomics analysis, although it was not possible to distinguish between SY and SE groups. We also applied global lipid profiling to plasma to further clarify the metabolic differences between each constitution type. The results were similar to the amino-acid-related metabolites; interestingly, the lipid profiles showed the possibility to distinguish the difference between the SE and SY groups $(p=0.06)$; thus, with a larger scale study, it may be possible to distinguish these two constitutions.

It has been reported that TE persons have a high prevalence rate of obesity due to hypoactivity of their energy metabolism [3, 22, 27]. The BMI of TE group was similar to that of the SY group, but the amino acids and lipid metabolites showed a significant difference between the TE and SY groups. Although the total cholesterol levels of the TE group were significantly higher than that of the SY group, they were within the normal range. The branched-chain amino acids, that is, lysine, tryptophan, cysteine, and glutamate, have been found to be present at high levels in obese individuals [28]. In our study, valine, glutamate, leucine, and lysine were more prevalent in the TE group than in the SE and SY groups, suggesting that metabolomics analysis can provide a scientific basis for explaining the proclivity of TE people to progress to obesity. Lysine and glutamic acid levels were also higher 


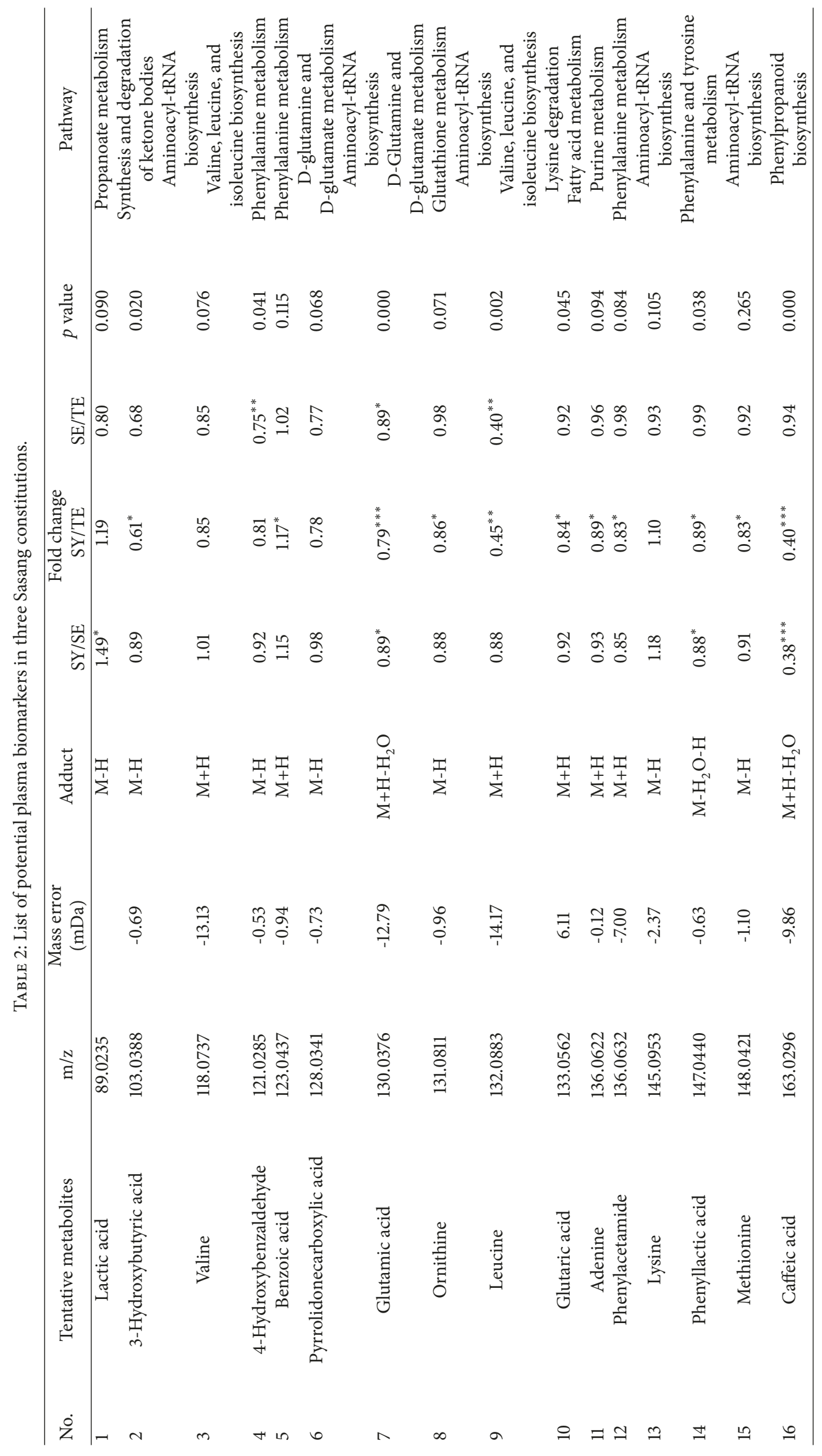




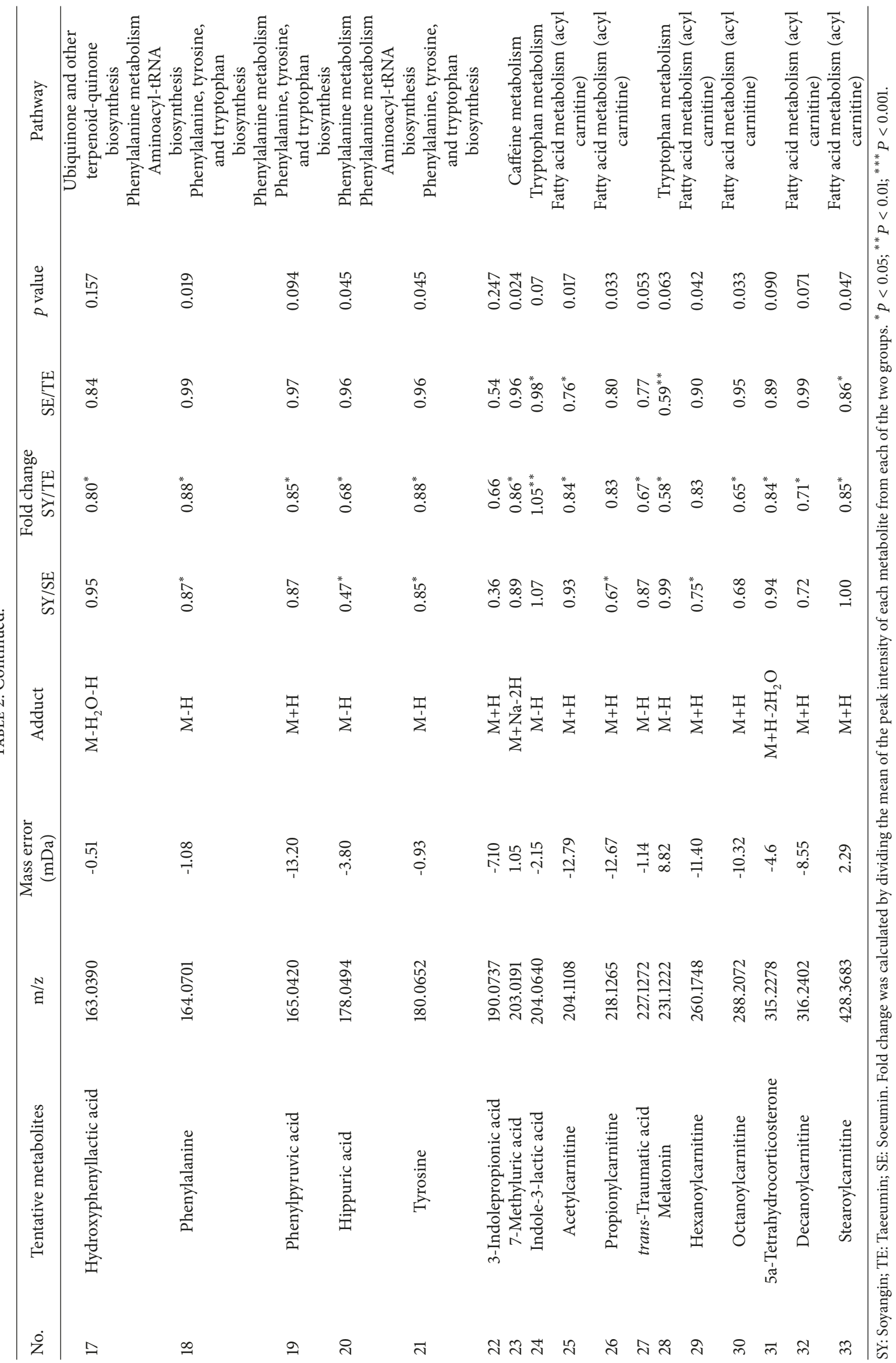




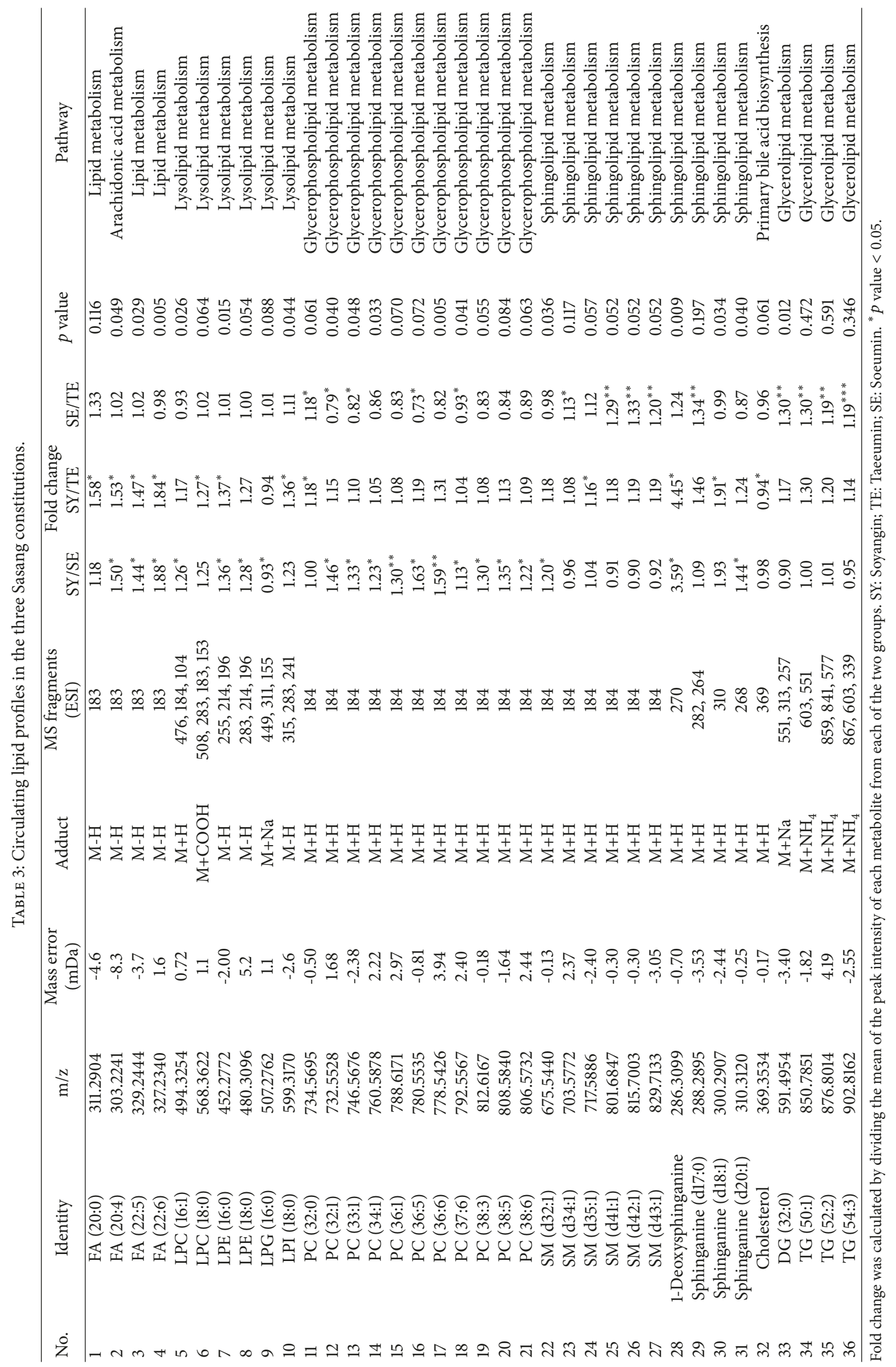


in the TE group. In particular, glutamic acid levels were significantly different among the three groups. Among the four Sasang types, TE persons had significantly higher total cholesterol and triglyceride (TG) values in the blood than other constitutions [3]. TE persons are more likely to be obese than other constitutions; however, our results showed that the BMI in the TE group was significantly different from that of the SE group but was not different from that of the SY group.

The concentration of lipid metabolites of the SY group was greater than that of the TE group. Levels of glycerophospholipids, lysolipids, glycerolipids, and shphingolipids differed significantly between each Sasang type, and levels were particularly higher in the SY group than in the TE groups. Low lipid concentrations may represent hypoactivity of lipid metabolism, and it is presumed that TE persons are more likely to be obese than an SY person with the same BMI; BMI is used to define obesity. Among the identified biomarkers, we found some interesting and compelling results: the level of 1-deoxysphinganine in the SY group was much higher than that in the other two constitution types. These levels are also elevated in diabetic patients and have been suggested to represent an early biomarker [29-31]. TE individuals have higher prevalence of diabetes than the other two types [32]. Since 1-deoxysphinganine is a toxic lipid for insulinproducing pancreatic $\beta$ cells [29], people with lower concentrations of the 1-deoxysphinganine can be more sensitive to this substance. In some circumstances, an increase in this substance in TE people may increase the incidence of diabetes mellitus rather than its incidence in SY people.

This study had some limitations. First, it involved a small sample size, which was inadequate to confirm the accuracy of the classification of the Sasang constitution type. Therefore, current evidence of metabolomics/lipidomics-based Sasang constitution medicine is insufficient to recommend classification according to constitution type. Second, the correlation between constitution and metabolite profiles in this study was based on healthy individuals in their twenties. So, it is not clear at this stage whether the metabolite profiles will hold across different age groups and health/disease conditions. In addition, since this study focused on male participants, further research is needed to identify gender differences in metabolites. One more limitation of this study is that it did not consider the environmental factors such as eating habit and lifestyle which might affect the results.

Collectively, the profiles of blood metabolites in TE and SY constitution types differ. Even when such individuals have the same BMI, it may thus be possible to distinguish between the two constitution types through metabolite analysis. Although this was a pilot study, the data revealed the possibility of diagnosis of the Sasang constitution type based on metabolomics and lipidomics analysis. Further clinical trials are required to verify these findings.

\section{Abbreviations}

BFP: $\quad$ Body fat percentage

BMI: $\quad$ Body mass index

PLS-DA: Partial least-squares discriminant analysis
QSCC II: Questionnaire for Sasang Constitution Classification II

SBP: $\quad$ Systolic blood pressure

SE: $\quad$ Soeumin

SY: Soyangin

TC: $\quad$ Total cholesterol

TE: Taeeumin

TG: $\quad$ Total triglyceride

VFA: Visceral fat area

WHR: Waist-to-hip ratio.

\section{Conflicts of Interest}

The authors declare that they have no conflicts of interest related to this study.

\section{Authors' Contributions}

Chang Hwa Jung wrote the manuscript and was responsible for study conception and design. Min Jung Kim and Da-Hye Lee performed the LC-MS analysis and interpretation of data. Jiyun Ahn, Eunju Do, and Tae-Youl Ha analyzed the data. All authors approved the final version of the manuscript.

\section{Acknowledgments}

The authors thank Professor MR Kim and AR Choi at Daegu Haany University's Daegu Oriental Hospital for supporting this investigation. This research was supported by Main Research Program of the Korea Food Research Institute (KFRI) funded by the Ministry of Science, ICT, and Future Planning.

\section{References}

[1] P. Gu and H. Chen, "Modern bioinformatics meets traditional Chinese medicine," Briefings in Bioinformatics, vol. 15, no. 6, pp. 984-1003, 2014.

[2] J. Yoo, E. Lee, C. Kim, J. Lee, and L. Lixing, "Sasang constitutional medicine and traditional Chinese medicine: a comparative overview," Evidence-Based Complementary and Alternative Medicine, vol. 2012, Article ID 980807, 17 pages, 2012.

[3] H. Chae, I. K. Lyoo, S. J. Lee et al., "An alternative way to individualized medicine: psychological and physical traits of Sasang typology," The Journal of Alternative and Complementary Medicine, vol. 9, no. 4, pp. 519-528, 2003.

[4] K.-H. Leem and H.-K. Park, "Traditional Korean medicine: now and the future," Neurological Research, vol. 29, supplement 1, pp. 3-4, 2007.

[5] J. Y. Kim and D. D. Pham, "Sasang constitutional medicine as a holistic tailored medicine," Evidence-Based Complementary and Alternative Medicine, vol. 6, supplement 1, pp. 11-19, 2009.

[6] S.-H. Kim, Y. Lee, B.-H. Koh, and E. Jang, "Assessing the diagnostic accuracy of the Questionnaire for Sasang Constitutional Classification II (QSCC II): A systematic review," European Journal of Integrative Medicine, vol. 5, no. 5, pp. 393-398, 2013.

[7] J.-H. Do, E. Jang, B. Ku, J.-S. Jang, H. Kim, and J. Y. Kim, “Development of an integrated Sasang constitution diagnosis method using face, body shape, voice, and questionnaire information," 
BMC Complementary and Alternative Medicine, vol. 12, article 85, 2012.

[8] S. H. K. B. Kim and I. B. Song, "A validation study of questionnaire of sasang constitution classification," Journal of Sasang Constitutional Medicine, vol. 5, no. 1, pp. 61-80, 1993.

[9] B.-Y. Kim, H.-J. Jin, and J. Y. Kim, "Genome-wide association analysis of sasang constitution in the Korean population," The Journal of Alternative and Complementary Medicine, vol. 18, no. 3, pp. 262-269, 2012.

[10] K. Sohn, A. Jeong, M. Yoon, S. Lee, S. Hwang, and H. Chae, "Genetic characteristics of Sasang typology: a systematic review," Journal of Acupuncture \& Meridian Studies, vol. 5, no. 6, pp. 271-289, 2012.

[11] M.-R. K. Shin and Dal-lae, "An Study on the Correlation between Sound Characteristics and Sasang Constitution by CSL," Journal of Sasang Constitutional Medicine, vol. 11, no. 1, pp. 137-157, 1999.

[12] D. S. Wishart, "Emerging applications of metabolomics in drug discovery and precision medicine," Nature Reviews Drug Discovery, vol. 15, no. 7, pp. 473-484, 2016.

[13] G. A. N. Gowda, S. Zhang, H. Gu, V. Asiago, N. Shanaiah, and D. Raftery, "Metabolomics-based methods for early disease diagnostics," Expert Review of Molecular Diagnostics, vol. 8, no. 5, pp. 617-633, 2008.

[14] World Health Organization, "Physical status: the use and interpretation of anthropometry. Report of a WHO Expert Committee," World Health Organization Technical Report Series, vol. 854, pp. 1-452, 1995.

[15] T.-G. Lee, B. Koh, and S. Lee, "Sasang constitution as a risk factor for diabetes mellitus: a cross-sectional study," EvidenceBased Complementary and Alternative Medicine, vol. 6, no. 1, pp. 99-103, 2009.

[16] D. Lee, M. J. Kim, E. Song et al., "Nutrikinetic study of genistein metabolites in ovariectomized mice," PLoS ONE, vol. 12, no. 10, article e0186320, 2017.

[17] J. M. Choi, T.-E. Kim, J.-Y. Cho, H. J. Lee, and B. H. Jung, "Development of lipidomic platform and phosphatidylcholine retention time index for lipid profiling of rosuvastatin treated human plasma," Journal of Chromatography B, vol. 944, pp. 157$165,2014$.

[18] M. Nam, M.-S. Choi, S. Jung et al., "Lipidomic profiling of liver tissue from obesity-prone and obesity-resistant mice fed a high fat diet," Scientific Reports, vol. 5, Article ID 16984, 2015.

[19] D. D. Pham, J. C. Lee, M. S. Lee, and J. Y. Kim, "Sasang types may differ in eating rate, meal size, and regular appetite: a systematic literature review," Asia Pacific Journal of Clinical Nutrition, vol. 21, no. 3, pp. 327-337, 2012.

[20] C. J.-H. Deok-Sang, L. Chang-Hoon, J. Jun-Bock, and L. KyungSub, "A study on reproducibility of responses to the questionnaire for sasang constitution classification II (QSCCII)," Journal of Korean Oriental Medicine, vol. 27, no. 3, pp. 145-150, 2006.

[21] Y. Kim, K. Park, J. Yoo, and E. Jang, "Sasang Constitution May Play a Key Role in Increasing the Number of SubElements of Metabolic Syndrome," The Journal of Alternative and Complementary Medicine, vol. 22, no. 3, pp. 204-211, 2016.

[22] E. Jang, Y. Baek, K. Park, and S. Lee, "Could the Sasang constitution itself be a risk factor of abdominal obesity?" BMC Complementary and Alternative Medicine, vol. 13, pp. 72-77, 2013.

[23] J. Um, J. Lee, J. Joo et al., “Association Between Tumor Necrosis Factor- $\alpha$ Gene Polymorphism and Sasang Constitution in
Cerebral Infarction," American Journal of Chinese Medicine, vol. 33, no. 04, pp. 547-557, 2005.

[24] N. Embade and O. Millet, "Molecular determinants of chronic liver disease as studied by NMR-Metabolomics," Current Topics in Medicinal Chemistry, vol. 17, no. 24, pp. 2752-2766, 2017.

[25] E. G. Armitage and A. D. Southam, "Monitoring cancer prognosis, diagnosis and treatment efficacy using metabolomics and lipidomics," Metabolomics, vol. 12, no. 10, article no. 146, 2016.

[26] A. M. McKillop and P. R. Flatt, "Emerging applications of metabolomic and genomic profiling in diabetic clinical medicine," Diabetes Care, vol. 34, no. 12, pp. 2624-2630, 2011.

[27] E. B. Shim, S. W. Lee, J. Y. Kim, C. H. Leem, and Y. E. Earm, "Taeeum-type people in Sasang constitutional medicine have a reduced mitochondrial metabolism," Integrative Medicine Research, vol. 1, no. 1, pp. 41-45, 2012.

[28] C. Takashina, I. Tsujino, T. Watanabe et al., "Associations among the plasma amino acid profile, obesity, and glucose metabolism in Japanese adults with normal glucose tolerance," Journal of Nutrition and Metabolism, vol. 13, article 5, 2016.

[29] R. A. Zuellig, T. Hornemann, A. Othman et al., "Deoxysphingolipids, novel biomarkers for type 2 diabetes, are cytotoxic for insulin-producing cells," Diabetes, vol. 63, no. 4, pp. 1326-1339, 2014.

[30] A. Othman, M. F. Rütti, D. Ernst et al., "Plasma deoxysphingolipids: A novel class of biomarkers for the metabolic syndrome?" Diabetologia, vol. 55, no. 2, pp. 421-431, 2012.

[31] M. Bertea, M. F. Rütti, A. Othman et al., "Deoxysphingoid bases as plasma markers in diabetes mellitus," Lipids in Health and Disease, vol. 9, article 84, 2010.

[32] N. H. Cho, J. Y. Kim, S. S. Kim, S. K. Lee, and C. Shin, "Predicting type 2 diabetes using Sasang constitutional medicine," Journal of Diabetes Investigation, vol. 5, no. 5, pp. 525-532, 2014. 


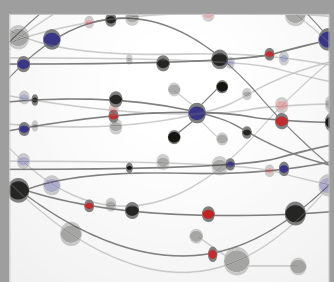

The Scientific World Journal
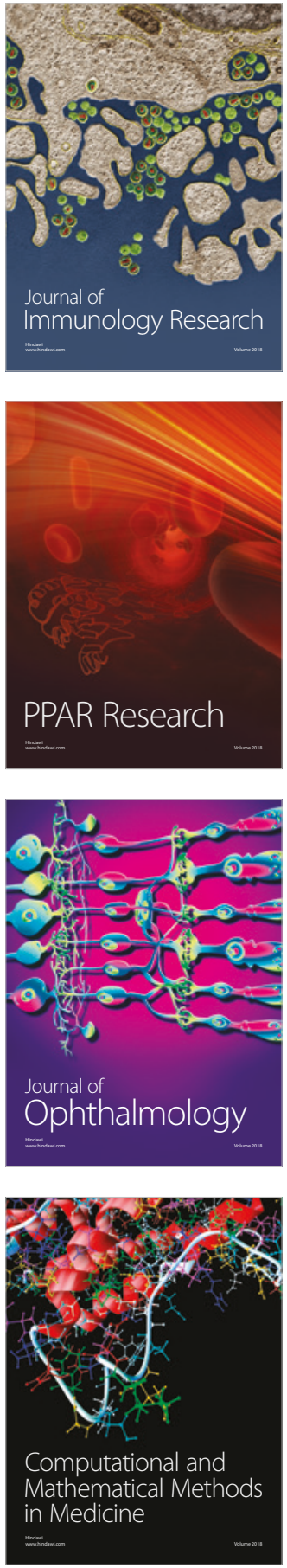

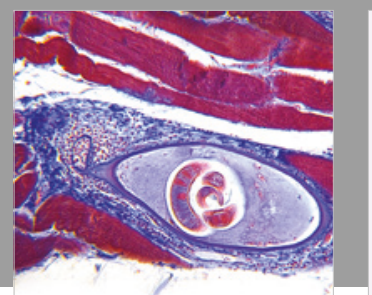

Gastroenterology Research and Practice

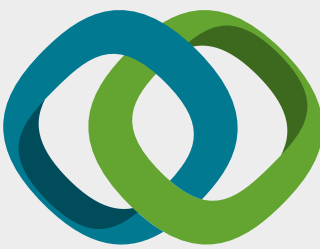

\section{Hindawi}

Submit your manuscripts at

www.hindawi.com
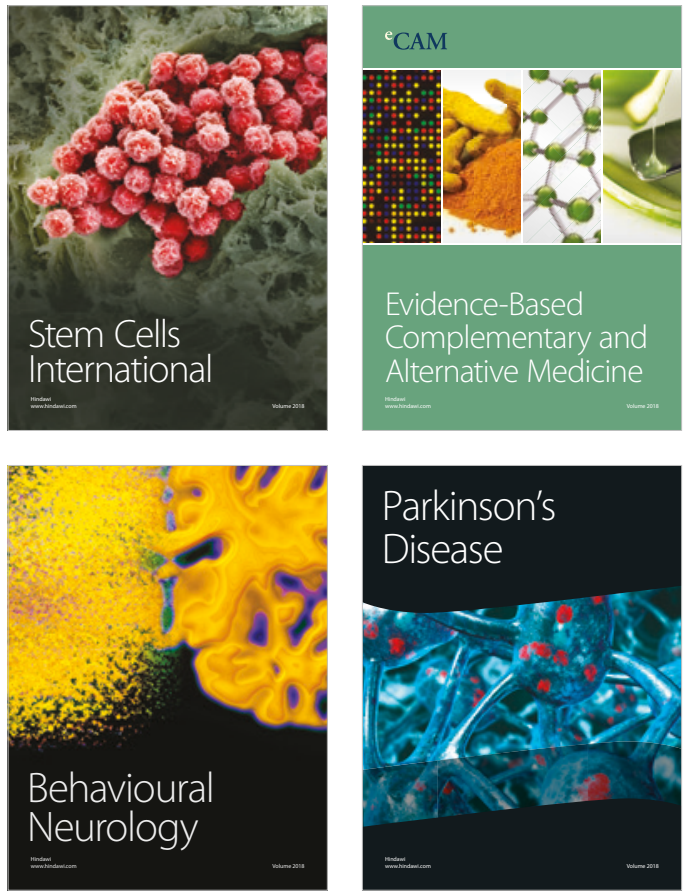

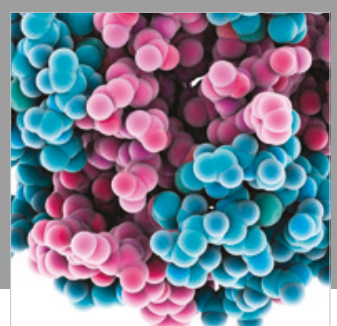

ournal of

Diabetes Research

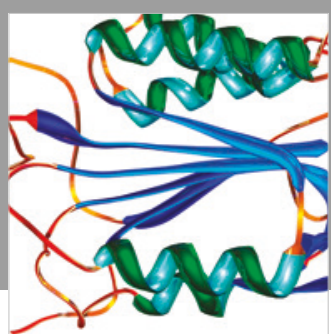

Disease Markers
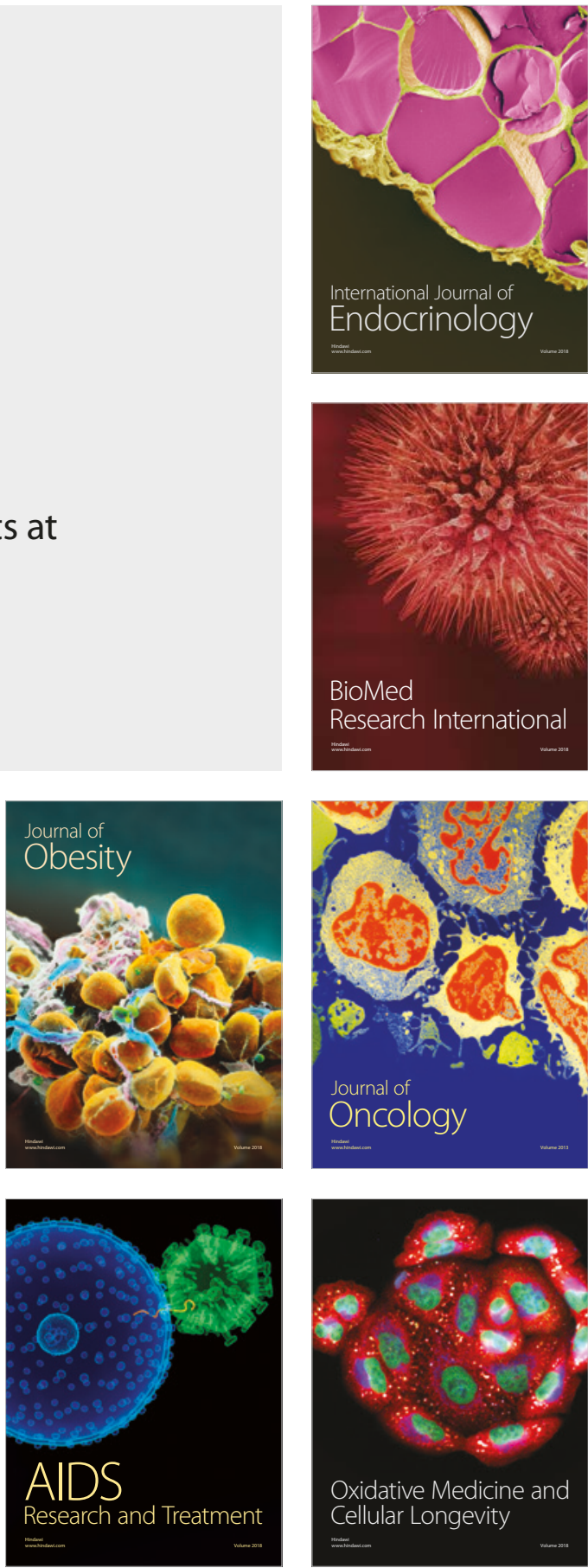